\title{
Magnetic Field Effect on Picosecond Electron Transfer
}

\author{
P. Gilch, F. Pöllinger-Dammer, C. Musewald, \\ M. E. Michel-Beyerle,* U. E. Steiner
}

\begin{abstract}
The recombination dynamics of a transition metal redox system monitored by femtosecond pump-probe spectroscopy are shown to be sensitive to high magnetic fields at times shorter than 10 picoseconds. The effect, based on coherent population beats of different spin states, is quantitatively accounted for and allows direct access to spin relaxation rates far beyond the time resolution of the fastest electron paramagnetic resonance technique. The presence of this ultrafast magnetic field effect helps in understanding complex reaction schemes in transition metal chemistry, which occur in a wide range of fields, such as bioinorganic chemistry and catalysis.
\end{abstract}

Magnetic field effects on the kinetics of radical pair (RP) recombination processes are well-established phenomena in molecular photochemistry (1). These effects are based on the principle of spin conservation in elementary chemical reactions and on the hyperfine and Zeeman interactions that affect the spin states of the RP. The hyperfine interaction in organic RPs induces transitions between the RP's spin states (one singlet $S$ and three triplet states $T_{+}, T_{0}$, and $T_{-}$). In an external magnetic field the Zeeman splitting removes the degeneracy of two triplet levels $\left(T_{+}\right.$and $\left.T_{-}\right)$with the singlet state, thus reducing singlet-triplet mixing (2). The saturation field of such effects is determined by the magnitude of the hyperfine interaction, which is $\sim 0.005$ $\mathrm{T}\left(1 \mathrm{~T}=10^{4}\right.$ gauss $)$ in organic RPs. This field range also determines the time scale for these effects to evolve, which is $\sim 10 \mathrm{~ns}$ $(3,4)$. In this regime, magnetic field effects have been successfully used to separate spin processes and elementary chemical processes such as electron transfer (ET) and bond formation (5-7).

Here, we report an ultrafast magnetic field effect on the recombination of an RP containing a transition metal component that occurs in less than $10 \mathrm{ps}$ in fields of several teslas. Using femtosecond pumpprobe spectroscopy and an optical superconducting magnet ( 8 ) we investigated the $\mathrm{RP}$ generated from ethylferrocene $(\mathrm{Fc})$ and oxazine 1 in its first excited singlet state $\left({ }^{1} \mathrm{Ox}^{+*}\right)$ in acetonitrile solution (9) at 298 $\mathrm{K}$. Analogous to the closely related $\mathrm{N}, \mathrm{N}$ dimethylmethylferrocene-methylene blue photo-redox system $(10),{ }^{1} \mathrm{Ox}^{+*}$ accepts an

P. Gilch, F. Pöllinger-Dammer, C. Musewald, M. E. Michel-Beyerle, Institut für Physikalische und Theoretische Chemie, Technische Universität München, D-85748 Garching, Germany. U. E. Steiner, Fakultät für Chemie, Universität Konstanz, D-78434 Konstanz, Germany.

*To whom correspondence should be addressed. Email: mibe@zentrum.phys.chemie.tu-muenchen.de electron from the neutral Fc. At high concentrations of $\mathrm{Fc}$, the forward ET measured in stimulated emission from ${ }^{1} \mathrm{Ox}^{+*}$ is ultrafast (time constant $\sim 200 \mathrm{fs}$ ) and follows essentially monoexponential kinetics. For energetic reasons the resulting RP $\left(\mathrm{Ox}^{\bullet} \cdot \cdot \mathrm{Fc}^{+\bullet}\right)$ can only recombine to form the singlet ground states of the reactants.

The recombination monitored by the recovery of $\mathrm{Ox}^{+}$shows multiexponential kinetics in zero field (Fig. 1). The dominant process proceeds with a time constant of $\sim 1 \mathrm{ps}$, a sizeable fraction decays with a time constant of $\sim 13 \mathrm{ps}$, and a small, longlived contribution has a characteristic time of $\sim 130 \mathrm{ps}$ that is responsible for the offset in Fig. 1. This rather complex behavior can be attributed to the transition metal component $\left(\mathrm{Fc}^{+\bullet}\right)$ in the RP. Replacing it by the cation of $\mathrm{N}, \mathrm{N}$-dimethylaniline results in a nearly monoexponential decay independent of magnetic fields (Fig. 2).

Magnetic fields of several teslas affect the recombination of the $\mathrm{Fc}^{+} \bullet$-containing RP (Fig. 1). The general effect at short times is to slow down the recombination. With increasing magnetic field the effect increases and its onset moves to earlier times. For the largest field applied, the magnetic field effect reaches its maximum at $2 \mathrm{ps}$. At longer times a cross-over behavior is observed, implying that, in contrast to the behavior at short times, increasing magnetic fields accelerate the recombination.

Qualitatively, the observed effects are accounted for by the following reaction scheme:

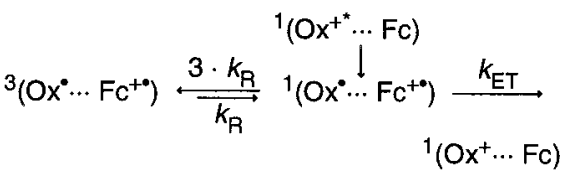

Generation of the RP by the singlet precursor ${ }^{1}\left(\mathrm{Ox}^{+*} \cdot \mathrm{Fc}\right)$ yields a singlet-correlated $\mathrm{RP}^{1}\left(\mathrm{Ox}^{\bullet} \cdot \mathrm{Fc}^{+\bullet}\right)$, which can recombine (rate constant $k_{\mathrm{ET}}$ ) to form the groundstate reactants. Alternatively, the RP may 
be reversibly converted to its nonreactive triplet spin state ${ }^{3}\left(\mathrm{Ox}^{\bullet} \cdot \cdots \cdot \mathrm{Fc}^{+\bullet}\right)$, a process that is determined by the extremely fast paramagnetic relaxation of $\mathrm{Fc}^{+} \bullet$, for which the (longitudinal and transversal) spin relaxation time $\tau_{\mathrm{s}}$ is on the order of a few picoseconds (11). The rate constant $k_{\mathrm{R}}$ characterizing the rate of multiplicity change in the RP corresponds to $1 /\left(4 \tau_{\mathrm{s}}\right)$. Thus, although the initial recombination rate constant of the RP is high, because of this stochastic spin process, the RP multiplicity develops toward a $1: 3$ singlet:triplet equilibrium, and the overall recombination rate constant slows down.

A magnetic field splits the triplet levels. In contrast to the hyperfine-dominated spin processes in organic RPs, this splitting does not affect the paramagnetic relaxation, which is probably dominated by an Orbach-type spin-orbit coupling mechanism $(12,13)$. On the other hand, the magnetic field induces a $\Delta g$-dependent mixing of the $S$ and $T_{0}$ RP states, leading to coherent $S \leftrightarrow T_{0}$ transitions with an angular frequency of

$$
\omega=\frac{1}{\hbar} \Delta g \mu_{\mathrm{B}} B_{0}
$$

where $B_{0}$ is the magnetic induction, $\mu_{\mathrm{B}}$ is Bohr's magneton, and $\Delta g$ is the difference of the $g$ values of $\mathrm{Fc}^{+} \bullet$ and $\mathrm{Ox}^{\bullet}$. For the latter, $g$ is close to the value of the free electron, whereas for the former, $g$ is a strongly anisotropic tensor so that the actual $g$ value depends on the orientation of the axis of $\mathrm{Fc}^{+}$relative to the field, which leads to a distribution of $\Delta g$ values spanning a range between -0.7 and 2.4 (15). With $|\Delta g|$ on the order of 1 , Eq. 1 yields $S$ $\leftrightarrow T_{0}$ oscillation periods on the order of 1 ps in a magnetic field of $10 \mathrm{~T}$. Consequently, population beats of the RP states are expected to occur on the same time scale as the spin-allowed RP recombination process. Of course, these population beats will modulate the rate of recombination. Analogous kinetic effects on the nanosecond time scale or longer have been observed for the recombination of RPs in solution and proteins $(16-19)$.

For a quantitative simulation of the observed kinetics, we made use of the stochastic Liouville equation formalism as specified by Steiner and Bürßner $(14,20)$, which has been applied to ferricenium systems (21). Before this study experimental verification of the formalism was confined to reaction yield modulations. The experiments presented here allow for a timedependent test of this model. Its features are as follows (Fig. 3):

1) The RP is described by using a basis set of four effective spin states $S^{\prime}, T_{0}^{\prime}, T_{+}^{\prime}$, and $T_{-}^{\prime}$ wherein the effective spin is quantized along the main $g$ tensor axis of $\mathrm{Fc}^{+} \bullet$.
These states are mixtures of pure singlet and triplet configurations. The weights $p_{\mathrm{s}, i}$ of the singlet component of state $i$ can be deduced from the experimental $g$ tensor values of $\mathrm{Fc}^{+} \bullet(21)$. The values obtained are $p_{\mathrm{s}, S^{\prime}}=0.82, p_{\mathrm{s}, T_{0}^{\prime}}=0.18$, and $p_{\mathrm{s}, T_{+}^{\prime}}=0$.

2) Because of the spin-selectivity rule for
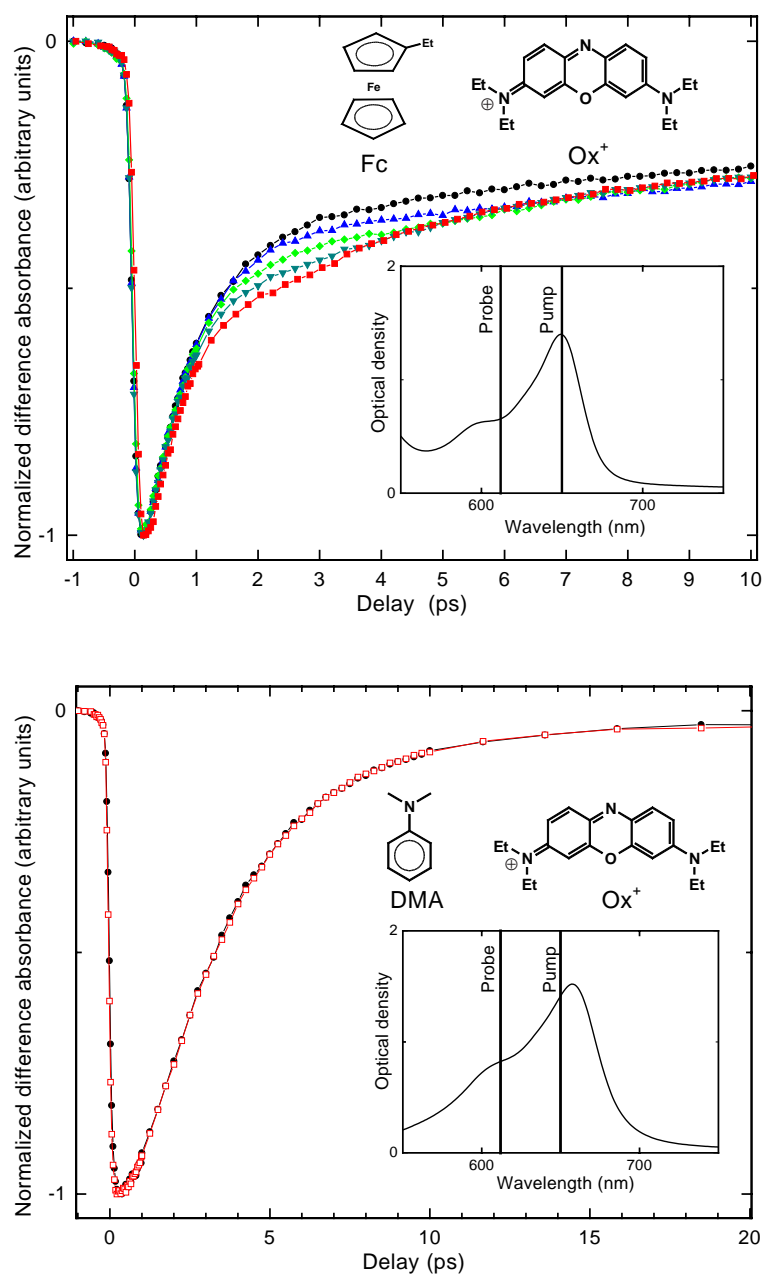

Fig. 2. Pump-probe measurements on the $\mathrm{N}, \mathrm{N}$-dimethylaniline (DMA)-oxazine 1 photo-redox system in magnetic fields of 0 (black) and 8.9 T (red). Pump and probe wavelengths as in Fig. 1.

Fig. 3. Magnetokinetic reaction scheme of the photo-redox system ethylferroceneoxazine 1 . The solid arrows indicate electron transfer processes (FET), the dashed arrows a relaxation transition between RP states. The diagrams display the time dependence ( 0 to $10 \mathrm{ps)}$ of the relative populations of the states involved in the recombination process, assuming a deltalike population of the RP

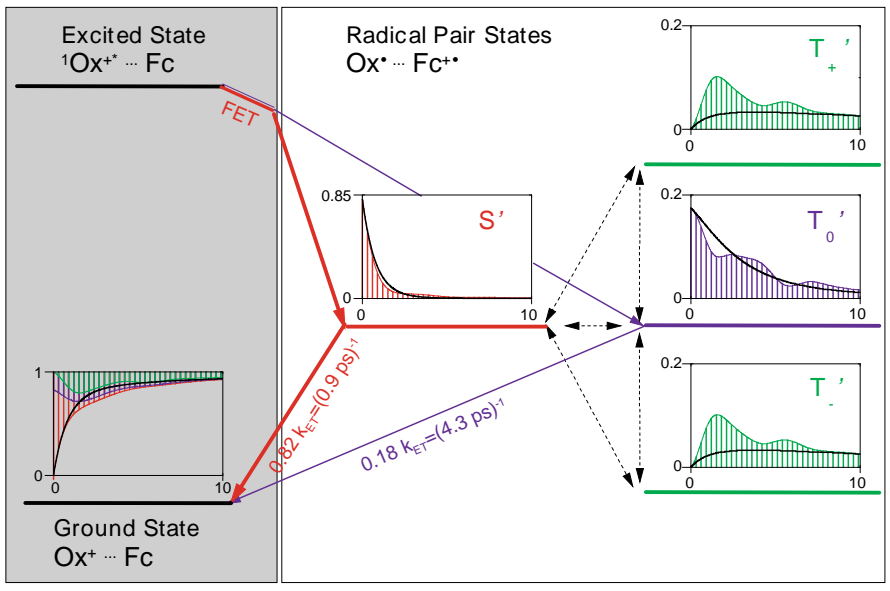
state. The time traces

refer to zero field (black lines) and $8.9 \mathrm{~T}$ (colored areas) and were simulated with best-fit parameters for the experimental data in zero field. The diagram marked ground state shows how the different RP states contribute to the time trace observed in the experiment at 8.9 T. 
3) The relaxation operator that describes relaxation among the four states is uniquely defined in terms of the field-independent paramagnetic relaxation time $\tau_{\mathrm{s}}$ of $\mathrm{Fc}^{+} \bullet$ as the only parameter.

4) The kinetic effect of an external magnetic field follows entirely from the action of the Zeeman Hamiltonian operator, which is determined by the $g$ tensors of the two radicals. It must be noted that because of the distribution of singlet character over the states, the recombination rate is not only modulated by $S^{\prime} \leftrightarrow T_{0}^{\prime}$ coupling but also by $T_{0}^{\prime} \leftrightarrow T_{+}^{\prime}$ and $S^{\prime} \leftrightarrow T_{ \pm}^{\prime}$ couplings.

At zero field, most of the RP population initially resides in $S^{\prime}$, which decays with a characteristic time of $\sim 1 \mathrm{ps,} \mathrm{mostly} \mathrm{to} \mathrm{the}$ ground states of the reactants but partially to the $T^{\prime}$ states. The decay of the latter either directly (in case of $T_{0}^{\prime}$ ) or indirectly via $S^{\prime}$ or $T_{0}^{\prime}$ (in case of $T_{+}^{\prime}$ ) is considerably slower.

For the maximum field of $8.9 \mathrm{~T}$, the calculated time profiles exhibit population beats corresponding to a net transfer of the population from reactive states to nonreactive and then back. These population beats cause the observed magnetic field effect of the recombination. The beat frequencies and the states coupled depend on the orientation of the $\mathrm{Fc}^{+} \bullet$ with respect to the field. A parallel $\mathrm{Fc}^{+} \bullet$ axis results in $S^{\prime} \leftrightarrow$ $T_{0}^{\prime}$ oscillations, whereas a perpendicular one yields $S^{\prime} \leftrightarrow T_{ \pm}^{\prime}$ and $T_{0}^{\prime} \leftrightarrow T_{ \pm}^{\prime}$ beats. Summation over all orientations is responsible for the wide range of Fourier components encountered here.

The experimental data were analyzed by
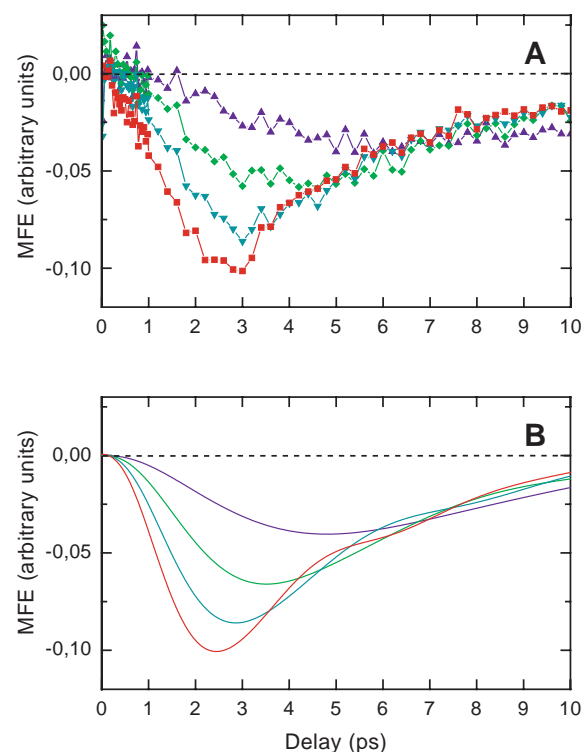

Fig. 4. Time dependence of the magnetic field effect (defined as the difference of the field time trace and the zero-field trace) for 3 (blue), 5 (green), 7 (cyan), and 8.9 T (red). (A) Experimental data. (B) Simulations as described in the text. fitting this model to the zero-field kinetics (22), the recombination rate constant $k_{\mathrm{ET}}$ and the spin relaxation time $\tau_{\mathrm{s}}$ being the only free parameters. These values can be used to predict the kinetics at arbitrary fields. Comparison of the predictions with the experimentally observed magnetic field effect (Fig. 4) shows almost quantitative agreement with respect to the amplitude of the magnetic field effect and the crossing feature at $\sim 7$ ps between the two sets of curves. This emphasizes the validity of the determined parameter values of $k_{\mathrm{ET}}$ and $\tau_{\mathrm{s}}$. We determined the value for the recombination rate constant $k_{\mathrm{ET}}$ to be $1.3 \mathrm{ps}^{-1}$, yielding ET lifetimes of $S^{\prime}$ and $T_{0}^{\prime}$ of 0.9 and $4.3 \mathrm{ps}$, respectively. The paramagnetic relaxation time $\tau_{\mathrm{s}}$ of $\mathrm{Fc}^{+} \bullet$ was determined to be $6.5 \mathrm{ps}$, which compares well with indirect results from nuclear magnetic resonance line-broadening measurements (11).

This observation of a magnetic field effect in ultrafast spectroscopy demonstrates that in RPs involving paramagnetic transition metal complexes, magnetic field effects can be observed on time scales three to four orders of magnitude shorter than in organic RPs. Moreover, this effect provides insight to the nature of the multiexponential kinetics resulting from the interplay between ET and spin processes. Thus, it provides a basis for accurate determination of ET rate constants and of paramagnetic relaxation times not measurable with paramagnetic resonance techniques. Furthermore, this spectroscopy might be used to identify RP-type intermediates in transition metal-containing reaction systems important in bioinorganic chemistry and in homogeneous catalysis.

\section{References and Notes}

1. See, for example, N. J. Turro, Modern Molecular Photochemistry (Benjamin Cummings, Menlo Park, 1978)

2. In the case of nonvanishing exchange interaction, the singlet-triplet mixing will pass through a maximum at a field matching this interaction.

3. M. E. Michel-Beyerle et al., Chem. Phys. 17, 139 (1976)

4. K. Schulten, H. Staerk, A. Weller, H.-J. Werner, B. Nickel, Z. Phys. Chem. Neue Folge 101, 371 (1976).

5. U. E. Steiner and T. Ulrich, Chem. Rev. 89, 51 (1989).

6. K. M. Salikhov, Y. N. Molin, R. Z. Sagdeev, A. L. Buchachenko, Spin Polarization and Magnetic Field Effects in Radical Reactions (Elsevier, Amsterdam, Netherlands, 1984).

7. M. Volk, A. Ogrodnik, M. E. Michel-Beyerle, in Anoxygenic Photosynthetic Bacteria, R. E. Blankenship, M. T. Madigan, C. E. Bauer, Eds. (Kluwer Academic, Dordrecht, 1995), pp. 595-626.

8. Femtosecond pump-probe measurements were performed with a Ti-sapphire laser system. The set-up and relevant pulse energies and durations are described in (10). Here, we restrict ourselves to the changes made to perform the field-dependent experiments. Pump and probe beams were crossed under a small angle in the center of a superconducting magnet. The propagation vectors of pump and probe beams were nearly perpendicular to the magnetic field; their polarizations were adjusted parallel with respect to each other and to the magnetic field in the measurements presented here. However, no influence of the relative polarization of pump and probe beam or pump and probe beam with respect to the field could be detected. For the recording of one time trace a certain field was set and kept constant during the measurements. By repeating the zero field time trace after a series of field measurements, we ensured the stability of the set-up and the sample.

9. Ethylferrocene was prepared from acetylferrocene (Aldrich) [R. A. Benkeser and J. L. Bach, J. Am. Chem. Soc. 86, 890 (1964)], purified by vacuum distillation, and characterized by nuclear magnetic resonance. Oxazine 1 (Lambda Physics) was laser grade, and acetonitrile (Merck) was spectroscopic grade. $\mathrm{N}, \mathrm{N}$-Dimethylaniline (Sigma) was distilled before use. The sample solutions $\left(1.1 \times 10^{-4} \mathrm{M}\right.$ oxazine 1 and $2.0 \mathrm{M}$ ethylferrocene in acetonitril; $1.8 \times 10^{-4} \mathrm{M}$ oxazine 1 in $\mathrm{N}, \mathrm{N}$-dimethylaniline) were measured in a 1-mm quartz glass cuvette.

10. P. Gilch, F. Pöllinger-Dammer, U. E. Steiner, M. E. Michel-Beyerle, Chem. Phys. Lett. 275, 339 (1997).

11. S. E. Anderson and R. Rai, Chem. Phys. 2, 216 (1973).

12. D. Kivelson, J. Chem. Phys. 45, 1324 (1966).

13. Rotational diffusion may also contribute to the relaxation of the $\mathrm{Fc}^{+} \bullet$ spin through spin-rotational coupling and anisotropic Zeeman interaction. In our model, which involves a semiempirical treatment of spin relaxation in a quasi-static isotropic distribution (14), these mechanisms are taken into account in an implict manner. An upper limit for their contribution can be deduced from the orientational correlation time of $\mathrm{Fc}^{+} \bullet$, which is $\sim 10$ ps (estimate based on the Debye relation).

14. U. E. Steiner and D. Bürßner, Z. Phys. Chem N.F. 169, 159 (1990).

15. For $g$ tensor values of ethylferricenium, the corresponding values of ferricenium were used because no experimental values are available for the former. However, monoalkyl substitution has little influence on the $g$ tensor, as demonstrated by the values of methylferrocenium [R. Prins and A. R. Korswagen, J. Organometal. Chem. 25, C74 (1970)]

16. A. V. Veselov, O. A. Anisimov, V. I. Melekhov, Y. N. Molin, Chem. Phys. Lett. 136, 263 (1987).

17. B. van Dijk, P. Gast, A. J. Hoff, Phys. Rev. Lett. 77, 4478 (1996).

18. S. Weber, E. Ohms, M. C. Thurnauer, J. R. Norris, G. Kothe, Proc. Natl. Acad. Sci. U.S.A. 92, 7789 (1995).

19. D. Stehlik, C. H. Bock, J. Petersen, J. Phys. Chem. 93 1612 (1989).

20. D. Bürßner, H.-J. Wolff, U. E. Steiner, Z. Phys. Chem. N. F. 182, 297 (1993).

21. P. Gilch, M. Linsenmann, W. Haas, U. E. Steiner, Chem. Phys. Lett. 254, 384 (1996).

22. Data analysis required deconvolution of experimental data of the experimental response function. The experimental response function was determined by two-photon absorption and modeled by a Gaussian function with a full width at half maximum (FWHM) of $180 \mathrm{fs}$. The experimental data were then fitted to a multiexponential function and an offset, both convoluted with the Gaussian function. The exponential terms were then taken as the deconvoluted experimental values that were fitted by the time-dependent solution of the stochastic Liouville equation by using the LevenbergMarquardt method. The simulation curves on which the difference curves in Fig. 4 are based were obtained by numerically convoluting the theoretical curves for delta function-type RP population process with the 180-fs FWHM Gaussian.

23. Financial support of the Deutsche Forschungsgemeinschaft and the Volkswagenstiftung is gratefully acknowledged. P.G. is indebted to the Fonds der Chemischen Industrie for a scholarship (KekuléStipendium). 